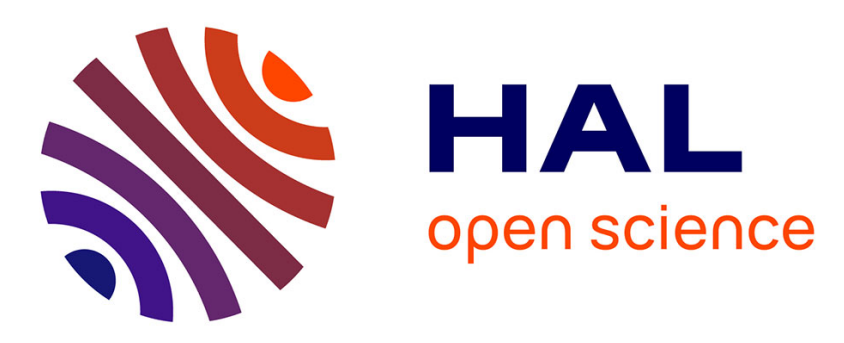

\title{
Tests of monolithic active pixel sensors at national synchrotron light source
}

\author{
G. Deptuch, A. Besson, G.A. Carini, D.P. Siddons, M. Szeleniak, M. Winter
}

\section{To cite this version:}

G. Deptuch, A. Besson, G.A. Carini, D.P. Siddons, M. Szeleniak, et al.. Tests of monolithic active pixel sensors at national synchrotron light source. Nuclear Instruments and Methods in Physics Research Section A: Accelerators, Spectrometers, Detectors and Associated Equipment, 2007, 570, pp.165-170. 10.1016/j.nima.2006.09.092 . in2p3-00133539

\section{HAL Id: in2p3-00133539 https://hal.in2p3.fr/in2p3-00133539}

Submitted on 7 Mar 2007

HAL is a multi-disciplinary open access archive for the deposit and dissemination of scientific research documents, whether they are published or not. The documents may come from teaching and research institutions in France or abroad, or from public or private research centers.
L'archive ouverte pluridisciplinaire HAL, est destinée au dépôt et à la diffusion de documents scientifiques de niveau recherche, publiés ou non, émanant des établissements d'enseignement et de recherche français ou étrangers, des laboratoires publics ou privés. 


\title{
Tests of Monolithic Active Pixel Sensors at National Synchrotron Light Source
}

\author{
G. Deptuch ${ }^{\mathrm{a},}$, A. Besson ${ }^{\mathrm{b}}$, G. A. Carini ${ }^{\mathrm{a}}$, D. P. Siddons ${ }^{\mathrm{a}}$, M. Szelezniak ${ }^{\mathrm{b}}$, M. Winter ${ }^{\mathrm{b}}$ \\ ${ }^{a}$ Brookhaven National Laboratory, Upton, NY 11973, USA \\ ${ }^{b} I P H C, C N R S-I N 2 P 3 / U L P, 23$ rue du Loess, BP 28, 67037 Strasbourg cedex 02, France
}

Elsevier use only: Received date here; revised date here; accepted date here

\begin{abstract}
The paper discusses basic characterization of Monolithic Active Pixel Sensors (MAPS) carried out at the X12A beam-line at National Synchrotron Light Source (NSLS), Upton, NY, USA. The tested device was a MIMOSA V (MV) chip, back-thinned down to the epitaxial layer. This $1 \mathrm{M}$ pixels device features a pixel size of $17 \times 17 \mu \mathrm{m}^{2}$ and was designed in a $0.6 \mu \mathrm{m}$ CMOS process. The X-ray beam energies used range from $5 \mathrm{keV}$ to $12 \mathrm{keV}$. Examples of direct X-ray imaging capabilities are presented.
\end{abstract}

Keywords: Monolithic Active Pixel Sensors; Synchrotron radiaton; X-ray imaging; Direct detection; Soft X-rays; Pixel detectors;

\section{Introduction}

Synchrotron radiation offers the advantages of high brightness and collimation with the additional capability of energy tunability through several types of monochromators. These intrinsic characteristics make it suitable for a wide range of applications.

The wavelength of the produced synchrotron radiation extends from infrared light to X-rays of hundreds of $\mathrm{keV}$ energy.

\footnotetext{
* Corresponding author.

tel.: +1 631344 2615; fax: +1 631344 5773;

e-mail: deptuch@ieee.org
}

Typically, light from high luminosity synchrotron radiation sources is used to reveal structures and features of individual atoms, molecules, crystals, cells and more. It was also used by condensed-matter physicists to investigate electrons surrounding the atomic nucleus and the position of atoms in molecules. New techniques are under development for medical diagnosis and the most recently for tumor therapy [1]. Being very intense and well collimated, it is generally preferred to radiation produced by conventional laboratory sources and offers a unique opportunity for characterizations on the nano-scale. Fast dynamic processes taking place in the samples can be efficiently studied due to the high luminosity of photon beams. Synchrotron radiation beams deliver high photon statistics in a short time 
providing good resolving quality for sequences of dynamic images.

Each experiment, performed at synchrotron facilities, requires a specific set of detectors satisfying some criteria, as for example image resolution, speed of the response, efficiency for a given energy range, and also price. Construction of a new generation of light sources requires the parallel development of new detectors. Detectors are elements which nowadays limit the exploitation of some challenging experiments.

One of the discussed ideas is the use of Monolithic Active Pixel Sensors (MAPS) for X-rays in the range from a few $\mathrm{keV}$ to about $10 \mathrm{keV}$. Due to its thin active layer (an epitaxial layer having thickness typically below $20 \mu \mathrm{m}$ ), the detection efficiency of standard MAPS detectors drops abruptly even below $10 \mathrm{keV}$. However, one can speculate about fabrication of MAPS detectors with thicker epitaxial layer if their usefulness for synchrotron radiation experiments is demonstrated.

Originally, MAPS were developed for visible light imaging [2]. Later they were introduced and successfully tested as single charged particle detectors for particle tracking in High Energy Physics $[3,4]$. They provide high spatial resolution and can be thinned down to reduce material budget or to allow backside illumination.

This paper discusses basic characterization of Monolithic Active Pixel Sensors (MAPS) in the X12A beamline at NSLS at BNL, USA. The tested device was a bottom thinned version of the MIMOSA V ${ }^{1}$ (MV) chip. The chip was operated at room temperature during the tests. The X-ray beam energies used range from $5 \mathrm{keV}$ to $12 \mathrm{keV}$. Examples of direct X-ray imaging capabilities are shown in the paper.

\section{Main features of MIMOSA V chips}

The detailed description of the MV chip, used in the tests at NSLS, was reported elsewhere [5]. Here only a brief description of the MV chip parameters

\footnotetext{
${ }^{1}$ MIMOSA V designed and fabricated in LEPSI-IReS collaboration, Strasbourg, France.
}

and the test set-up is given. This $1 \mathrm{M}$ pixel device features pixel size of $17 \times 17 \mu^{2}$ and it was designed in a $0.6 \mu \mathrm{m}$ CMOS process. The device used in the tests was a bottom-thinned chip. The thickness of the active layer, remaining after thinning, was $10 \mu \mathrm{m}$. The expected detection efficiencies are about $45 \%$ and $5 \%$ for X-ray energies $5 \mathrm{keV}$ and $12 \mathrm{keV}$, respectively (from the limits of the range used). The readout clock frequency was $10 \mathrm{MHz}$, resulting in the integration time equal to about $25 \mathrm{~ms}$. The processing of data, sent out of the MV chip as analogue signals from sequentially addressed pixels, relied on Correlated Double Sampling (CDS). The result was calculated as a difference of whole, successively acquired frames. The CDS operation was implemented in hardware in FPGA on the data acquisition board [6]. Subtraction was performed after digitization. The result of CDS was sent to a PC using the USB 2.0 connection. The chip was mounted on a small printed circuit board equipped with analogue drivers connected with the acquisition board by a flat cable.

The MV chip was tailored to the detection of single relativistic charged particles. Back-thinning was used to allow detection of low energy electrons in another experiment [5]. The parameters of the detector, particularly the dynamic range, were not optimized for the environment of the X-ray beam. Integration of signals over multiple frames was necessary to provide enough statistics without saturation in single frames.

\section{Tests at NSLS}

The PC board with detector was installed in a light-tight Al box. A square hole in a box in front of the detector was covered with a thin mylar foil. The detector was installed on the stage allowing programmable movements in vertical and horizontal directions perpendicular to the X-ray beam. The height of the beam size spot was too small to cover the whole surface of the detector. Typically, a synchrotron emitted photon beam has a rectangular shape with the horizontal side longer. During the experiments the beam was resized through a V-ladder system down to the size of about $0.3 \times 9 \mathrm{~mm}^{2}$ outspreading horizontally. Thus, the vertical scanning 
was necessary in order to obtain the image using a whole active area of the detector. The scan was achieved by moving the stage during the time when the beam was on and the acquisition of image frames was running continuously. The step motors were programmed to move the stage by a fixed distance and to stop at each position to allow acquisition of some number of frames. The imaged specimen was fixed on the box in front of the detector and moved together with the stage. The final images, presented in this paper, were obtained after some software processing. Basically, all acquired frames were summed up. The algorithm allowing selection of the exposed strips from each image was used in order to avoid adding noise from dark parts of the image from each step. Since the steps were slightly overlapping, the transition between steps was smoothed by averaging the amplitudes in the overlapped parts. The beam intensity was tuned up to avoid saturation of pixels by placing appropriate number of layers of $\mathrm{Al}$ foil in the beam path.

\subsection{Imaging of a bone specimen with a Ti implant}

The first imaged specimen was a piece of a bone with a Ti wire implant inside. The form of the specimen was cuboid with the base of about $5 \times 5 \mathrm{~mm}^{2}$. The thickness of the Ti wire was about $0.4 \mathrm{~mm}$. It was installed in a bone leaving some gap between the wire and the bone substance. The view of the imaged specimen is shown in Fig. 1. Such interface gaps may develop in the region of a bone implants causing an implant failure. They may exist also upon insertion. Capability of imaging such gaps is important for medical diagnosis and is the subject of studies and development of new imaging techniques requiring the use of synchrotron radiation [7]. The images were taken at the X-ray energy of $8.2 \mathrm{keV}$.

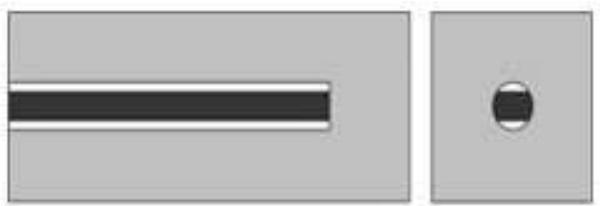

Fig. 1. View of the cuboid specimen of the bone with Ti implant inside; implant thickness is about $0.4 \mathrm{~mm}$, there is a small gap between the bone substance and the implant.
The first image, presented in Fig. 2, was obtained for the specimen positioned with two walls parallel to the detector plane. The radiograph shows a uniform density of the bone substance, the dark part reflecting the Ti wire, and the lighter outline of the gap between the wire and the bone. The second image, shown in Fig. 3, was obtained for the bone specimen placed in such a way that the walls were inclined about $45^{\circ}$ to the detector plane. The gradient of intensity due to the increasing thickness of the imaged bone substance towards the center of the sample is visible.

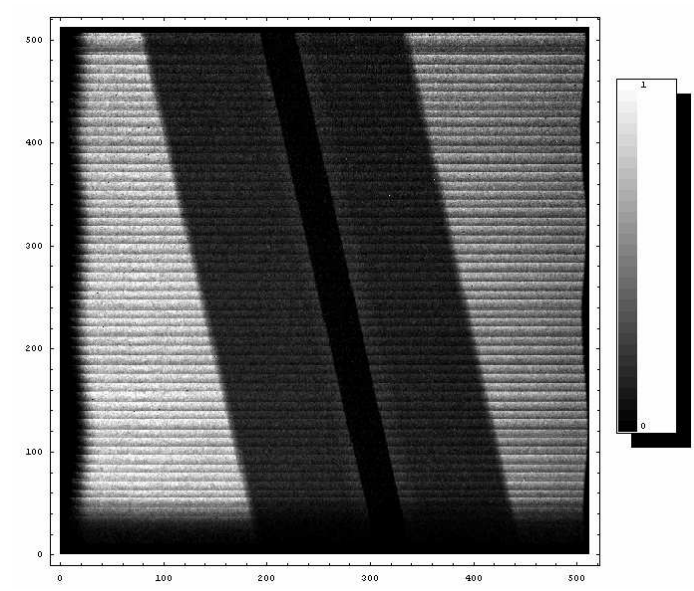

Fig. 2. Image of the cuboid bone sample with Ti implant with two walls of the sample oriented parallel to the detector plane taken at $8.2 \mathrm{keV}$ X-ray energy.

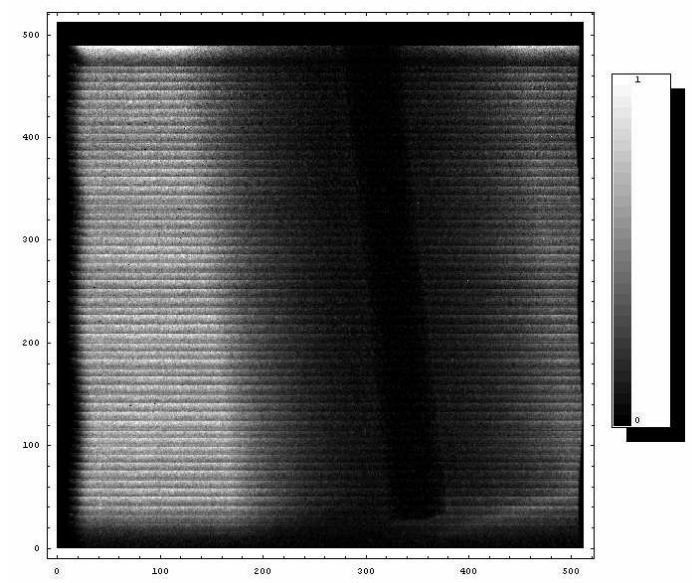

Fig. 3. Image of the cuboid bone sample with Ti implant with walls of the sample inclined $45^{\circ}$ to the detector plane taken at $8.2 \mathrm{keV} \mathrm{X}$ ray energy. Gradient of intensity due to the increasing thickness of the substance towards the center of the specimen is visible. 
The estimated number of photons in the lightest parts of both images is about 200 photons/pixel. The sample of the bone with an implant was used for imaging upon availability of the specimen. The imaging presented in this paper was not part of the studies of the mentioned particular effort toward the development of imaging techniques of bone-metal gaps. However, the results are very attracting since the tiny gap is clearly visible in the image.

\subsection{Example of cockchafer radiograph}

Another example of the radiography image is shown in Fig. 4. A small cockchafer was chosen as an object due to the presence of components like: soft tissue, chitin shell, antennas, etc., giving different contrast.

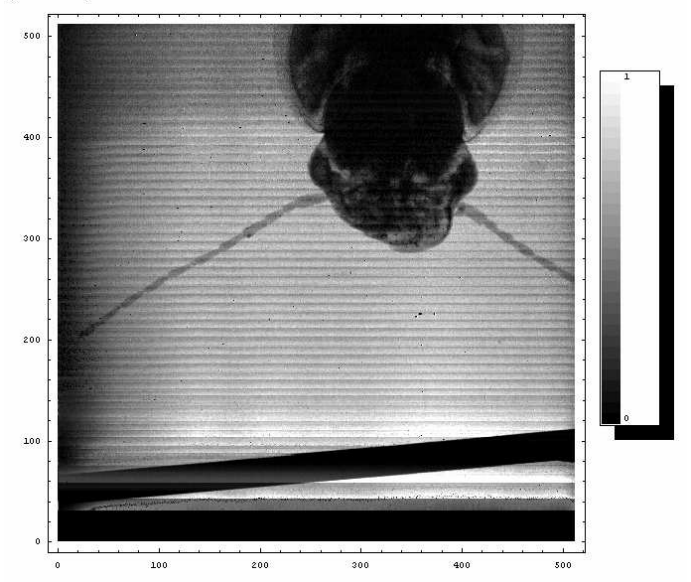

Fig. 4. Radiograph of the cockchafer taken at $5.2 \mathrm{keV}$ X-ray energy.

The energy of the X-ray beam was $5.2 \mathrm{keV}$. The dark trace at the bottom of the image is a $\mathrm{W}$ wire used to orient the scanning.

\subsection{Resolving of multiple photon incidents}

MAPS are signal integrating detectors. The charge originating from multiple impacts falling within one integration time (equal to the readout time for the MV chip) is integrated on the pixel capacitance and then the resulting voltage is read out. In the case of the detector characterized by a very low readout noise and fully efficient charge collection, the number of incident photons from a monoenergetic beam can be deduced from the signal magnitude. The readout noise of MAPS detectors is very low typically between $10 \mathrm{e}^{-}$and $25 \mathrm{e}^{-}$[8]. On the other hand, the charge collection based on thermal diffusion causes charge spreading between neighboring pixels and some charge losses in the charge collection process. The number of incident photons within one integration time is governed by Poisson statistics. The goal of this exercise was to check the limit at which number of incident photons individual impacts cannot be distinguished due to overlapping of corresponding peaks in the spectra.

A collimator, as shown in Fig. 5, with a pin hole of about $10 \mu \mathrm{m}$ was used. The beam intensity was adjusted, by inserting $\mathrm{Al}$ foils, to the desired range from less than one up to above 10 photons falling within single integration time. The beam energy was set to $12 \mathrm{keV}^{2}$.

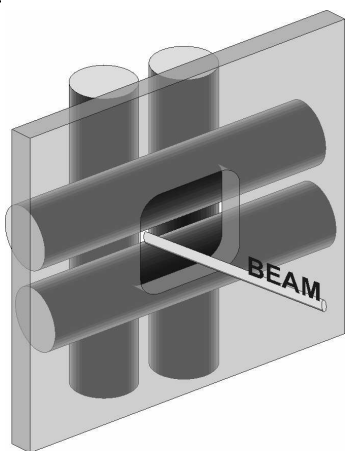

Fig. 5. $10 \mu \mathrm{m}$ hole collimator used in the experiment made of four W cylinders.

The collected data were treated with the dedicated data analysis software. Only a sub-window of the size $26 \times 26$ pixels was analyzed. The sub-window was centered on the beam impact position. The pedestals were calculated from an empty pedestal data acquisition run and subtracted later on fly. Single $12 \mathrm{keV}$ photons produced signals of about 150 $\mathrm{ADCU}^{3}$. A cut on signals larger than $25 \mathrm{ADCU}$ allowed efficient noise suppression. An image,

\footnotetext{
${ }^{2}$ lower energy resulted in the undesired presence of $3^{\text {rd }}$ order harmonics that were seen by the detector.

${ }^{3}$ ADCU - analog-to-digital conversion units, the gain of the detector was not accurately calibrated; the conversion gain was about $0.075 \mathrm{ADCU} / \mathrm{e}^{-}$.
} 
integrating all hits found in 4100 frames acquired, is shown in Fig. 6. The most of the signal of photons passing through the pin hole is concentrated in the central point of the image.

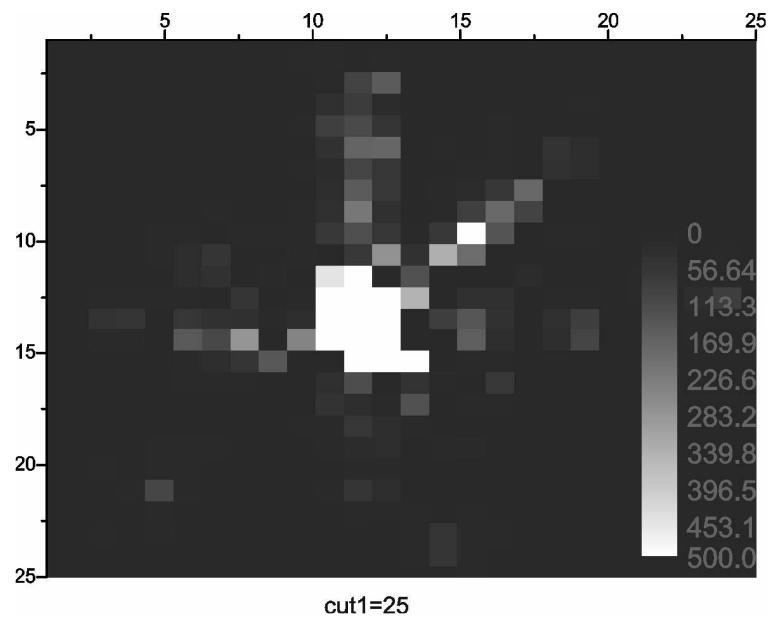

Fig. 6. $26 \times 26$ pixel sub-window with all identified hits projected on a common plane; view of the collimator taken at $12 \mathrm{keV} X$-ray energy.

There are some photon impacts, around this point. Selecting only impacts (clusters of $5 \times 5$ pixels with signal) around the center point, a spectrum for impacts of single photons was built. The histogram is shown in Fig. 7. The peak position is about $150 \mathrm{ADCU}$.

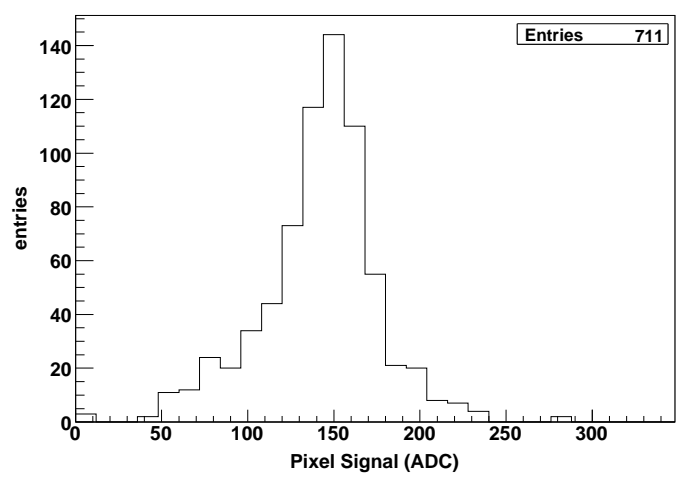

Fig. 7. Spectrum of $12 \mathrm{keV}$ photons for a cluster of $5 \times 5$ pixels obtained from single photon impacts outside the collimator spot.

Then, varying the beam intensity and considering only signals from pixels directly in front of the pin hole, the spectra for multiple photons impacts were built. The examples of the histograms obtained are shown in Fig. 8 for average less than 1 photon per integration time and in Fig. 9 for about 10 photons falling within one integration time.

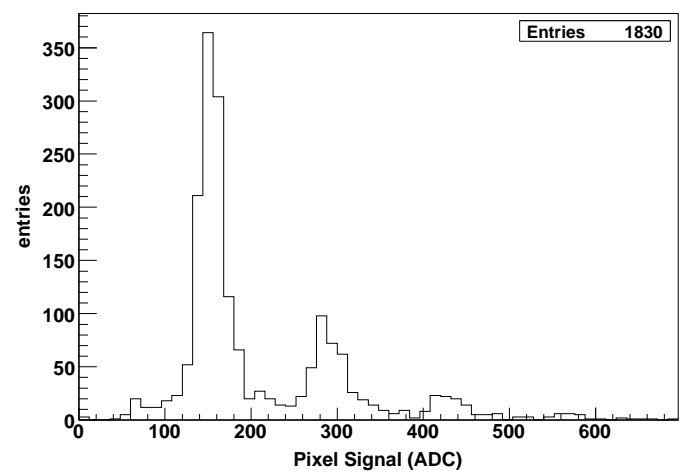

Fig. 8. Spectrum of multiple impacts of photons (average less than one photon per single acquisition) for $12 \mathrm{keV} \mathrm{X-rays} \mathrm{for} \mathrm{a} \mathrm{cluster}$ of $5 \times 5$ pixels from photon impacts inside the collimator spot.

Histograms feature multiple peaks, resulting from multiple photon conversions on the targeted pixel per frame readout time according to Poisson statistics.

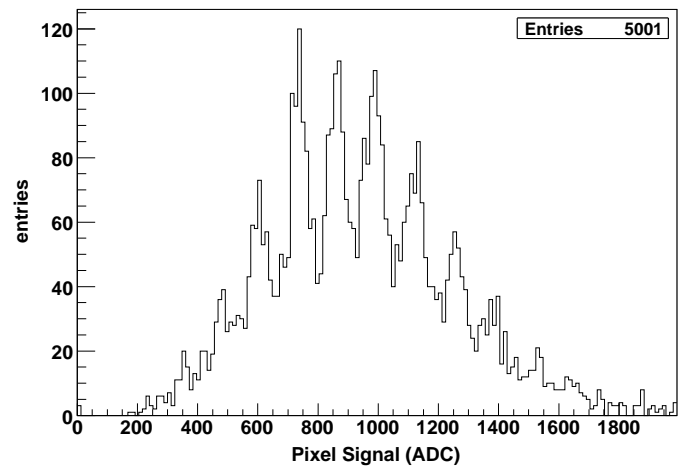

Fig. 9. Spectrum of multiple impacts of photons (average about 10 photons per single acquisition) for $12 \mathrm{keV}$ of $\mathrm{X}$-rays for a cluster size of $5 \times 5$ pixels from photon impact inside the collimator spot.

The response of the detector to the impact of more than one photon is a convolution of responses to single photons. A simple Monte Carlo analysis, allowing simulation of the spectrum resulting from the convolution of multiple single photon spectra, was used to verify the results obtained in tests. The spectrum of single photons from measurements was 
used to define probability density function and to produce the results of convolution. The simulated spectrum for average of 10 photons converted during one integration time is shown in Fig. 10. The simulated and measured spectra are very similar. When increasing average number of photons per frame above 10 , the separate peaks started to be indistinguishable, smeared in the wide background.

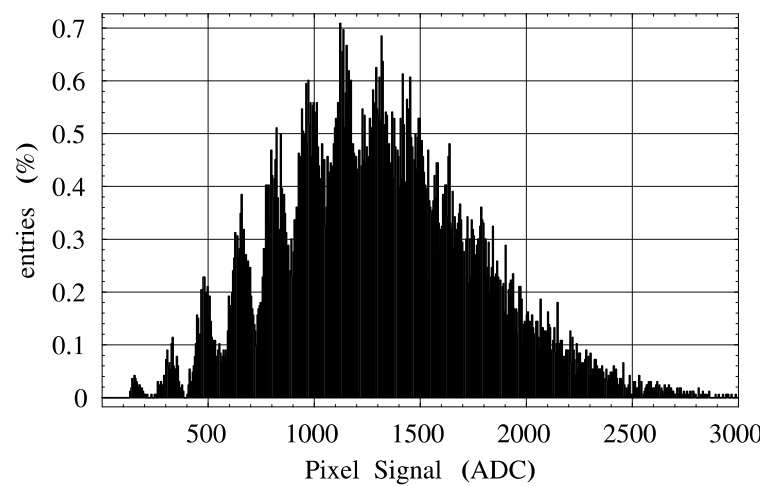

Fig. 10. Simulated spectrum of multiple photons impacts, obtained by convolution of a single photon spectra respecting Poisson statistics (average 10 photons per single acquisition) for a cluster size of $5 \times 5$ pixels.

\section{Conclusions}

The construction of a new generation of synchrotron light sources triggers new development of detectors. MAPS detectors may be considered as an option for some imaging tasks, especially where high 2-D resolution is required. They can be used as direct X-ray imagers. With current state MAPS detectors, the range of $\mathrm{X}$-ray energies for imaging is limited to soft X-rays to about $10 \mathrm{keV}$. Due to the thin active layer, which is an epitaxial layer having thickness below $20 \mu \mathrm{m}$, the detection efficiency of standard MAPS detectors drops abruptly even below $10 \mathrm{keV}$. However, fabrication of MAPS with thicker epitaxial layer is technologically possible but this option may mean a non standard fabrication process and higher cost. The newest proposals for radiation detectors postulate fabrication of monolithic structures by wafer bonding which can be an appealing alternative to standard MAPS devices but also at higher cost.
The paper shows examples of high resolution Xray radiography images and studies of resolving multiple photon impacts. Despite thin active layer, high sensitivity of the detector allowed contrasts in images to show tiny elements in the imaged objects. The work is considered as an initial study of usefulness of MAPS detectors for experiments using synchrotron radiation. It is worth highlighting the fact that the tested device was not optimized in any degree for the use in any experiment involving synchrotron radiation

Significant X-ray fluxes hit an imaging detector during experiments. The tested device was not designed following guidelines for the radiation hardness nor was the chip designed in a new submicron CMOS process that offers intrinsically better radiation resistance than older technologies. Thus the radiation hardness was not addressed in the current work. Radiation hardness of MAPS has been addressed in literature and is the subject of undergoing studies [10]. The radiation resistance up to the levels of $30 \mathrm{Mrd}$ has been reported [11]. This experience may be the guideline for the design of MAPS dedicated to experiments with high X-ray fluxes.

\section{Acknowledgments}

The authors wish to thank Wolfgang Caliebe and Zhong Zhong. Use of the National Synchrotron Light Source, Brookhaven National Laboratory, was supported by the U.S. Department of Energy, Office of Science, Office of Basic Energy Sciences, under Contract No. DE-AC02-98CH10886. This manuscript has been authored by Brookhaven Science Associates, LLC under Contract No. DEAC02-98CH10886 with the US Department of Energy. The United States Government retains, and the publisher, by accepting the article for publication, acknowledges, a world-wide license to publish or reproduce the published form of this manuscript, or allow others to do so, for the United States Government purposes. 


\section{References}

[1] F.A.Dilmanian, Z.Zhong, T.Bacarian, H.Benveniste, P.Romanelli, R. Wang, et al., "Interlaced X-ray microplanar beams: A radiosurgery approach with clinical potential", PNAS, vol. 103, n0. 25, pp.9709-9714, 2006

[2] E.R.Fossum, "CMOS image sensors: Electronic Camera-OnA-Chip", IEEE Trans. Electron Devices, vol. ED-44, pp.16891698,1997

[3] R.Turchetta, J.D.Berst, B.Casadei, G.Claus, C.Colledani, W.Dulinski, et al., "A momolithic active pixel sensor for charged particle tracking and imagingusing standard VLSI CMOS technology", Nucl. Instrum. Meth. A 458, (2001), 677-689

[4] G.Deptuch, J.D.Berst, G.Claus, C.Colledani, W.Dulinski, Y.Gornushkin, et al., "Design and Testing of Monolithic Active Pixel Sensors for Charged Particle Tracking", IEEE Trans. Nucl. Sci. vol. 49, No. 2, pp. 601-610, 2002

[5] G.Deptuch, W.Dulinski, M.Caccia, M.Winter, "High Resolution, Back-Side Illuminated Monolithic Active Pixel Sensor for Low-Energy Electron Imaging", IEEE Trans. Nucl. Sci. vol. 53, No. 5, pp. 1745-1754, 2005

[6] A. Czermak, et al., "Data Acquisition System for Silicon Ultra Fast Cameras for Electron and Gamma Sources in Medical Appications (Sucima imager), in Proceedings of the Seventh International Conference on Advanced Technology and Particle Physics, Villa Olmo, Como, 15-19 Oct. 2001

[7] D.M.Connor, D.Sayers, D.R.Sumner, Z.Zhong, "Diffraction enhanced imaging of controlled defects within bone, including bone-metal gaps", Phys. Med. Biol. 51, pp. 3283-3300, 2006

[8] G.Deptuch, G.Claus, C.Colledani, M.Deveaux, W.Dulinski, "Development of Monolithic Active Pixel Sensors for charged Particle Tracking", Nucl. Inst. and Meth. A 511, pp. 240-249, 2003

[9] V.Suntharalingam, R.Berger, J.A.Burns, Ch.K.Chen, C.L.Keast, J.M.Knecht, "Megapixel CMOS image sensor fabricated in three-dimensional integrated circuit technology", Proceedings of IEEE SSCC, pp. 356-357, 2005

[10] M.Deveaux, J.D.Berst, W.de Boer, M.Caccia, G.Claus, G.Deptuch, et al. "Charge collection properties of X-ray irradiated monolithic active pixel sensors", Nucl. Inst. and Meth. A 552, pp. 118-123, 2005

[11] El-Sayed, et al. "Design and characterization of ionizing radiation-tolerant CMOS APS image sensors up to $30 \mathrm{Mrd}(\mathrm{Si})$ total dose", IEEE Trans. Nucl. Sci., vol. 48, pp. 1796-1806, 2001 